Voix et Images

volxetimages

\title{
Idéologie et poésie : un poème de Paul-Marie Lapointe
}

\section{Michel Biron}

Volume 14, numéro 1 (39), automne 1988

France Théoret : narratrice de la subjectivité

URI : https://id.erudit.org/iderudit/200755ar

DOI : https://doi.org/10.7202/200755ar

Aller au sommaire du numéro

Éditeur(s)

Université du Québec à Montréal

ISSN

0318-9201 (imprimé)

1705-933X (numérique)

Découvrir la revue

Citer cet article

Biron, M. (1988). Idéologie et poésie : un poème de Paul-Marie Lapointe. Voix et Images, 14(1), 90-118. https://doi.org/10.7202/200755ar d'utilisation que vous pouvez consulter en ligne.

https://apropos.erudit.org/fr/usagers/politique-dutilisation/ 


\title{
Idéologie et poésie: un poème de Paul-Marie Lapointe
}

\author{
par Michel Biron, Université de Montréal
}

En 1963, dans le second numéro d'une toute nouvelle revue montréalaise appelée Parti pris, paraît pour la première fois le poème «Psaume pour une révolte de terre» de Paul-Marie Lapointe. L'éditorial de ce numéro s' intitulait «La révolution et la morale» et opposait très clairement deux types de morale: la morale révolutionnaire proposée par le F.L.Q. ${ }^{1}$ et la Morale, l'hypocrite et l'inhumaine, celle que pratiquent les représentants de l'ordre établi. Le premier article du numéro était signé Paul Chamberland et portait sur «Aliénation culturelle et révolution nationale». Pour le lecteur de Parti pris, il ne fait pas de doute que le mot «révolte» dans le titre du poème prolonge le mot «révolution» qu'il retrouve presque à chaque page de la revue. La revue se donne à lire comme une matrice de textes divers mais homogènes d'où se dégage un certain consensus idéologique. La plate-forme idéologique de Parti pris est connue: Nous luttons pour un Etat libre, lä̈que et socialiste ${ }^{2}$. Avant même d'être lu, le poème de Lapointe profite ou est affecté d'un vecteur de sens préalable: l'imprimatur partipriste oriente la lecture qu'on peut en faire. "Psaume pour une révolte de terre», poème indépendantiste, laïcisant et socialiste? En un sens, aberrant il est vrai, le poème admet par sa seule inscription dans l'espace paginal de la revue ces trois étiquettes idéologiques. Mais le poème ainsi phagocyté par le discours partipriste n'est pas celui qu'on lit au tout début du recueil Pour les âmes ${ }^{3}$. Transcrit sur un papier qui n'appartient plus à la revue, le même poème se manifeste autrement à la lecture. Il apparaît maintenant qu'il ne se satisfait pas de tous les lieux idéologiques de Parti pris. Mais comment s'écrit le différend du poème avec ce discours? Que fait le poème de ces lieux idéologiques? Il les déplace, les réaménage, les modalise? Il les transpose, transforme, transgresse? Les conteste, les nie, les abolit?...

\section{Le sens obvie du poème}

«Psaume pour une révolte de terre» se présente globalement comme une prière pour l'appropriation d'un espace (terre, fleuves), d'un corps (étalon, mère) et de l'âme. Reportons l'analyse du mot «psaume» à plus loin parce qu'elle

1 Front de libération du Québec: nom que se sont donnés différents groupes terroristes québécois à partir de 1963.

2 «Présentation», Partl pris, vol. I, $n^{\circ} 1$, octobre 1963, p. 4.

3 Le Réel absolu. Pòmes 1948-1965, Montréal, l'Hexagone, coll. «Rétrospectives», 1971,270 p. 
forcerait à anticiper sur la seconde partie de cet article et arrêtons-nous pour commencer au sens obvie du poème: "pour une révolte de terre». La révolte n'est pas seulement située au niveau de la terre, elle est constituée de terre, comme si celle-ci devenait la matière même de la révolte. Terre: atterrées (p. 207), terreau (p. 208), terreur (p. 208), terre propice (p.210); le mot ne représente pas seulement la matière, il devient lui-même, en tant que signifiant, la matière sonore du poème. Et le paradigme peut se prolonger si l'on pense à des expressions comme terre-mère ou terre à terre qui correspondent tout à fait à l'univers sémantique du poème. Mais «terre» se lit d'abord par opposition à «ciel», à l'inaccessible: l'absolu céleste devient dans Pour les âmes un absolu terrestre, habitable, chaleureux. On lit par exemple dans «Courtes pailles»:

\author{
divinité embusquée \\ entre les pierres cachée \\ qui tombe de l'arbre comme pomme \\ ou surgit ô geyser et soudain \\ en un siècle quelconque \\ autrefois ou demain \\ vous saisit a la gorge
}

mystère de la terre (p. 229).

Ce parti pris pour le terrestre, pour le concret, confere un privilège aux objets de tous les jours, aux choses familières, à la matière simple, au corps. Lapointe inscrit son poème dans l'ordre répétitif du quotidien, du «au jour le jour», du travail toujours recommencé.

L'homme, dans les premiers mots du poème, est dépossédé: nul amour n'a la terre qu'il embrasse / et ses fleuves le fuient. La négation absolue, le ton péremptoire, le présent atemporel des verbes et la forme brève mais achevée du distique rappellent le modèle de l'aphorisme. Parole d'affirmation et de vérité, l'aphorisme refuse de se justifier, refuse d'être justifié, incontestable par définition et saturé de sens. Des Maximes de La Rochefoucauld aux formules concises et "performantes» de Valéry et de Char, l'enjeu est le même: combiner un maximum de sens dans un minimum de mots. Cette plénitude du sens sert cependant à exprimer la désappropriation la plus totale puisque l'espace se retire comme s'il allait être hors de la portée humaine. Mais hors de portée de qui, de quoi? De l'amour? Oui, si l'on considère «amour» comme la figure métonymique de l'amoureux. Cet enfouissement du sens dans la couche rhétorique du texte est d'autant plus frappant qu'il se produit dans l'espace par excellence de la transparence, du sens obvie, c'est-à-dire dans l'aphorisme.

Le poème s'ouvre ainsi, du moins en ce qui concerne son contenu, dans l'ordre de la négativité. Celle-ci est confirmée à la strophe suivante dont le point d'appui est le substantif isolé «colère»:

colère

diluvienne métamorphose 
tes blessés reposent en délire

tes paroles sont vides et les jours se répètent (p. 207).

Le substantif isolé, qu'on pense par exemple au poème «Arbres» ${ }^{4}$, est l'élément privilégié du discours poétique de Lapointe. Ici encore, «colère» apparaît soudainement, hors de toute actualisation (article, pronom), suivi par d'autres substantifs. La colère, sentiment propre à la révolte, déclenche une succession contigué de syntagmes laconiques. Les images de blessés, de sang et de mort soulignent la violence de la révolte, mais c'est en fin de compte le sentiment de futilité qui domine. Réaction à la vanité de la parole et du geste humains, la révolte de terre est menée par les sentiments (colère, amour) plutôt que par la raison. Le chaos, ce monde sans loi pour l' homme $e^{5}$, posé comme un donné de la réalité en ce début de poème, découle du fait que tout semble échapper à la maîtrise de l'homme. L'emploi de deux pronoms possessifs à la deuxième personne (tes blessés, tes paroles) ne fait qu'ajouter au désordre signifié un désordre syntaxique puisque ces pronoms ne se rapportent pas à une personne, mais à un sentiment, métonymiquement relié à une personne il est vrai: ce sont les blessés et les paroles de la colère, c'est-à-dire de l'homme en proie à la colère. La très grande liberté des énoncés les uns par rapport aux autres produit un effet d'accumulation qui accélère le rythme et accentue le sentiment d'une perte de contrôle:

la mise à mort est parée de dentelles

et le sang gicle dans la foule

maternités futiles (p. 207).

Mais le chaos des premières strophes fait sens. Négativité ne veut pas dire anarchie totale et gratuite: cela signifie en termes clairs que la colère propre à la révolte est provoquée par une situation intenable où le discours est vain et où la mort n'est rien de plus qu'une dentelle, une décoration. Dans l'imaginaire révolutionnaire, la mort $n$ 'a de sens que si elle est utile: mourir, c'est faire avancer la cause révolutionnaire, c'est un sacrifice nécessaire, l'acte tragico-héroïque auquel s'accroche tout le sens de la révolution. Une révolution sans morts, c'est une révolution tranquille...

Quelle est la situation intenable qui provoque la colère? La terre et les fleuves ne définissent aucun lieu particulier, l'espace décrit est de dimension cosmique. Le premier indice topologique relativement précis est «la foule» (cité plus haut). La foule n'est évidemment pas un lieu, mais elle se définit

4 Arbrell'arbre est clou et croix/croix de rail et de papier (p. 174). Voici ce qu'écrit Nepveu à propos du substantif dans «Arbres» : Le substantif, par l'omission de tout article et de tout procès, prend une valeur absolue, ce qui entraîne par contrecoup la substantivation de l'adjectif lorsque celui-ci est isolé par le blanc (les Mots a l'écoute. Poésie et silence chea Fernand Ouellette, Gaston Miron et Paul-Marie Lapointe, Québec, Presses de l'université Laval, 1979, p. 233).

5 Paul-Marie Lapointe, «Foi en l'homme», cité par Guy Robert, Poésie actuelle. Littérature du Québec, Montréal, Librairie Déom, 1970, p. 85. 
doublement comme l'espace de l'indifférenciation des individus et, métonymiquement, comme la rue dans la ville (la foule se tient dans la rue). L'image de la foule assistant à la mise à mort évoque peut-être les exécutions sur la place publique qui, même disparues depuis longtemps des mœurs judiciaires, continuent à frapper l'imaginaire, chaque révolte se mirant dans une sorte d'invariable révolutionnaire: le mythe de la mise à mort publique. On peut se demander à cet égard si la pierre et le feu dont il est question entre parenthèses ne sont pas justement les instruments de l'insurrection citadine. En deuxième référence topologique se lit le mot «usine» (prend l'usine, p. 207) qui comporte à l'évidence le sème «travail» et la reprise du sème "ville» déjà rencontré dans «foule». L'organisation du travail dans la ville industrielle décrit de manière déjà plus nette le type de situation figuré dans le poème. En troisième lieu, le lecteur apprend que les pères vont aux bois le dimanche (p. 207). «Bois» s'oppose à «ville» comme «dimanche» s'oppose à «semaines» (semaines perpétuelles et vaines, p. 207). Ce qui est mis en évidence ici, c'est la constitution du calendrier hebdomadaire qui oblige le travailleur à s'échiner six jours pour ne «vivre» que le seul jour qui lui reste. Première constatation: l'horaire de vie qu'on impose au travailleur est inacceptable et inhumain.

La division du travail à laquelle est assujetti le travailleur rend celui-ci amorphe, aliéné et le pousse peu à peu vers la mort: périssent les hommes et les jours au jour le jour (p. 208). Le temps qui fuit devient le temps mort. Ce vers peut se lire sur les modes subjonctif et indicatif, comme une sentence, un arrêt du destin ou comme un constat froid et distancié. L'inversion de la phrase permet de doubler la répétition du mot «jour», superposant de la sorte la redondance phonétique à la redondance sémantique. Il se crée une sorte d'envoûtement sonore par lequel le travailleur semble être pris au piège, tenu par le mouvement monotone du temps. De plus, cette inversion permet de remonter le cours habituel de la lecture. La phrase se joue de son rythme habituel, modifie le cours normal du temps de lecture là où s'exprime paradoxalement son irréversibilitét. La figure de l'inversion sera exploitée deux autres fois dans le poème, et dans chaque cas, le vers admet le sème du temps qui passe: entre les îles circulent des glas des cargos (p. 209) reprend l'image récurrente du fleuve comme métaphore de la fuite des choses (Héraclite) et passent les vies (p. 209) se réfere directement à l'inéluctable passage du temps. À force de révoquer ce qu'il présente sous le signe de l'irrévocable, le poème tend à nier la force linéaire de l'écoulement temporel. Ce faisant, il cherche, sans jamais y parvenir, à neutraliser le temps.

Tout le recueil de Lapointe est obsédé par l'idée de la répétition. Pierre Nepveu déclare que Pour les âmes est le recueil du temps perdu, le temps répétitif du quotidien 7 . L'homme est «quotidien», rongé par l'invisible douleur du temps amorphe; il n'aperçoit guère devant lui qu'un éclat de temps, un jour à la fois,

6 Le groupe MU note ce renversement paradoxal dans le vers d'Apollinaire: Sous le pont Mirabeau coule la Seine. Il écrit: Inverser la phrase même où s'exprime la fuite du temps, c'est une tentative sans espoir pour en remonter le cours (Rhétorique de la poesse. Lecture linéaire, lecture tabulaire, Bruxelles, Ed. Complexe, 1977, p. 144-145). 
quelques heures peut-être. Mais le quotidien est dénoncé au nom d'un quotidien meilleur, plus humain, plus vivable. Lapointe ne cherche donc pas à sortir de la quotidienneté, mais à la racheter en lui adjoignant des valeurs qui ne lui sont pas reconnues traditionnellement: dignité, liberté, profondeur... Comme la terre, qui désigne plus que la terre dans le poème (elle est absolue), le quotidien ne se réduit pas au routinier (il récupère l'énergie de la pérennité).

Cette transformation du «au jour le jour» dégradé par un calendrier meurtrier en un quotidien gratifiant est aussi lisible dans le discours de Parti pris. D'ailleurs, plusieurs traits du poème mentionnés jusqu'ici caractérisent également le discours partipriste. Dans un paragraphe de l'éditorial du second numéro que je cite en entier, plusieurs ressemblances rapprochent les deux discours de révolte. Il est question de la Morale politique des forces de l'ordre à l'occasion des procès du F.L.Q., des manifestations de la Place des Arts et d'une grève des débardeurs:

Cette Morale hypocrite et inhumaine, notre peuple la gobe depuis plus d' un siècle, mais aujourd' hui elle est enfin déconsidérée: la jeunesse est entrain (sic) d'en découvrir une autre, ramenée du ciel à la terre, de l'éternité d notre vie quotidienne, incarnée dans notre exigence de liberté et de dignité. Nous ne concevons de morale que celle qui se fonde sur les besoins et les aspirations de l'homme quotidien; et nous entendons créer une société où l'abolition de l'exploitation et de l'oppression coloniale rende possible l'assouvissement de cette aspiration à la dignité. En attendant, la seule morale qui tienne se fonde dans la réalité de la lutte révolutionnaire: est moral ce qui contribue d notre libération. 8

Plusieurs idées clefs passent d'un texte à l'autre. La référence au peuple indique bien quels sont les protagonistes de la révolte dans les deux cas. Parti pris écrit «l'homme quotidien», Lapointe écrit pauvres, travailleurs, petit homme, plongeuses de nuit, main-d'auvre... Les dénominations de Lapointe paraissent même plus restrictives que celle de Parti pris: il s'agit avant tout de l'ouvrier, dont la force physique («muscles», «sueur», «force», «reins», «mains») représente l'unique force productive. Paul Chamberland parlera de son côté de l'homme des besoins ou de l'homme concret ou encore de l'opprimé. Jamais le mot «prolétaire» n'apparaît dans ce numéro, ce qui est significatif si l'on considère l'orientation socialiste de la revue. Par ailleurs, l'espoir en la jeunesse sur laquelle mise Parti pris est évoqué par Lapointe, notamment dans le dernier distique: les fruits savent posséder l'âme / et l'étalon la mère. L'insistance des deux textes à fixer la révolte sur la terre et à régler les horloges à l'heure de la quotidienneté est remarquable. L'équipe de Parti pris cherche à se délester de l'héritage métaphysique et de l'humanisme abstrait qu'on a attribués, entre autres, à la revue Cité libre ${ }^{9}$. La volonté de transformer cette société basée

8 «Éditorial. La révolution et la morale», Partl pris, vol. I, n², novembre 1963, p. 3. Je souligne.

9 André-J. Bélanger écrit: Parti pris ne cesse de redire son opposition d l'homme abstrait, universel et citoyen du monde que Cité libre s'était fixé comme idéal (Ruptures et 
sur l'exploitation de la force de travail est manifeste dans les deux textes. La révolte ou la révolution ${ }^{10}$ prônées de part et d'autre supposent en somme un réaménagement social de la collectivité qui passe par l'abolition du système capitaliste.

Lire «Psaume pour une révolte de terre» comme une critique corrosive du capitalisme n'est pas très original (la poésie surréaliste a souvent été interprétée ainsi), mais je voudrais marquer de manière insistante les traces textuelles sur lesquelles cette lecture s'appuie. La négativité initiale du poème n'est pas étrangère aux conditions d'existence du travailleur. La récurrence du sème «travail» au sens le plus ouvriériste du terme est remarquable: usine, semaines, travailleurs, muscles, force, pain, plongeuses de nuit, soudées, reins, clou... Le travailleur est pauvre, il a faim, il a peur:

(mes villes aux pauvres pauvres dans l'aisselle des coffres mes agités

$$
\text { futiles }
$$

$$
\text { travailleurs) }
$$

et plus loin:

\author{
petit homme \\ irremplaçable petit homme \\ avec ta faim \\ et la terreur qui te fuit et te poursuit (p. 208).
}

Le temps mécanique abolit tout espoir de retour sur le passé, l'énergie exsudée est perdue pour de bon au profit des «coffres». Dans les mines (dans le métal des mines, p. 210), dans les champs (dans le fumier des récoltes, p. 210) et dans les forêts (dans les racines des parallèles, p.210), la situation est la même: nos os futiles reposent dans leurs chairs (p. 210). À qui renvoie le possessif «leurs»? «Leurs» s'oppose à «nos», «chairs» à «os», l'observation est clinique, glacée; entre les deux termes de l'opposition, entre eux et nous, que peut-on voir sinon un conflit entre deux classes sociales, avec d'un côté le charnu, le repu et de l'autre le squelette? Même le corps du travailleur ne lui appartient pas: il sert à engraisser l'autre.

L'enjeu de la «révolte de terre» n'est pas la victoire politique, ponctuelle et insuffisante. Car la lutte, précise Lapointe, renferme ses propres récompenses et

constantes. Quatre idéologies du Québec en éclatement: La Relève, la JEC, Ciué libre, Parti pris, Montreal, Hurtubise HMH, 1977, p. 161).

10 Lapointe confond à peu de choses près les deux termes: dans son article intitulé «Foi en 1'homme», il parie de la véritable Révolution en rappon avec le devenir politique des hommes et écrit deux lignes plus loin que le devoir de la poésie, sa raison est la Révolte. Le mot «Révolution» correspond apparemment à la traduction en langage politique de la révolte poétique. 
peut ne se résoudre qu' en ses défaites et victoires. ${ }^{11}$ La révolte, prise dans cette acception favorable de génératrice de vie $e^{12}$, n'a pas de fin: interminable colère si l'étoile chavire (p. 210). Or si l'étoile chavire, c'est-à-dire si l'étoile céleste se renverse et se transforme en une étoile de terre (on retrouve la nécessité de faire du bas l'espace global de la vie, ce qui n'exclut pas qu'il y ait de l'altitude, mais celle-ci se définit toujours en fonction du sol), alors le sentiment de colère associé à la révolte de terre n'aura plus de terme. Dans la longue séquence qui suit, cette colère est canalisée dans un militantisme explicite: si la colère piétine après l' heure du lunch pancartes en mains avec un cri et des armes. La révolte passe par l'arrêt de travail, la grève et le piquetage, voire l'affrontement direct et violent entre les ouvriers et les patrons.

La voix du patron est d'ailleurs clairement et ironiquement perceptible dans cette déclaration d'innocence: dieu sait pourquoi? nous les traitions comme un fils nous étions un père. Beau cas de dialogisme tout à fait bakhtinien, inscrit en plein cour du poème. On devine le commentaire que pourrait faire Bakhtine devant cet exemple: la parole cachée d'autrui, cette citation de l'opinion patronale, est une représentation, une caricature du langage d'une classe sociale pour qui toute perturbation de l'ordre établi est une infraction, une menace qu'il faut réprimer. La feinte naïveté de l'énoncé enchâssé accentue le confort et l'assurance du sujet qui l'exprime. Deux voix sont donc nettement audibles: la voix ironique du poète et la voix représentée du patron.

Il est remarquable que le langage soit ainsi stratifié en deux niveaux, mais, ajouterait Bakhtine, cela ne prouve d'aucune façon que le dialogisme au sens fort soit possible en poésie ${ }^{13}$. Lapointe représente dans cette tirade le style d'autrui qu'il isole entre deux tirets de sorte que l'énoncé ironique se trouverait, d'après Bakhtine, en dehors du langage direct du poète. Ce faisant, Lapointe se sert d'un procédé propre au roman, et ce n'est plus de la poésie «pure». Cette «prosaïsation» du langage poétique est justement ce qui permet au poète de polémiquer avec le langage des patrons. Ceux-ci sont pris à partie comme groupe social, dénoncés en tant que classe privilégiée qui tire profit du travail des ouvriers et qui parvient surtout à tenir un discours de générosité dans l'exploitation la plus inhumaine. En s'étonnant de la colère des petits du monde (p. 210), les patrons laissent entendre que s'il y a révolte, c'est à cause des révoltés et non du système dont ils se portent garants. Le poème révèle aux travailleurs leur propre

11 Paul-Marie Lapointe, «Foi en 1'homme», loc. cit., p. 85.

12 Ibid.

13 Pour avoir un aperçu plus précis de ce que Bakhtine appelle la prosaïsation du symbole poétique, se référer aux pages 148-151 et 403-408 d'Esthétique et théorle du roman. En voici un extrait significatif : Pour bien comprendre la distinction entre bisémie poétique et bivocalité prosaïque, il suffit de comprendre n'importe quel symbole et de l'accentuer de bout en bout de manière ironique (naturellement, dans un contexte important correspondant), autrement dit, d'y introduire sa propre voix, d'y réfracter son intention nouvelle. De ce fait, le symbole poétique (tout en restant symbole, s'entend) est transféré en méme temps au plan de la prose, devient discours bivocal : entre le discours et son objet s'insère un discours, un accent étranger, et sur le symbole tombe une ombre d'objectivation (naturellement, la structure bivocale se révélera primitive et simple). 
humiliation afin qu'ils cessent de porter avec culpabilité le poids de l'injustice sociale.

La poésie cherche-t-elle à rendre consciente de leur propre humiliation une classe d'hommes qui ne la liront jamais de toute façon? Pour débarrasser les travailleurs de ce sentiment misérabiliste, presque masochiste, le poème est-il efficace? Peu importe la réponse, constatons cependant qu'il s'agit d'un thème cher à la revue Parti pris. On exacerbe les oppositions de classe pour conjurer l'attitude soumise et humiliante de la classe laborieuse entretenue par les discours paternalistes des dirigeants. André-J. Bélanger résume la position de Parti pris:

Pour Parti pris le grand atout de l' «autre», c'est sans conteste d'être parvenu d nous faire refouler le ressentiment normal que nous pouvons nourrir contre lui, pour le retourner contre soi sous forme de culpabilité, c'est-d̀-dire haine de soi-même. Selon ce même processus psychologique nous aurions été conduits à renverser notre instinct de vie - désir de vie - en instinct de mort, névrose collective qui se traduit par une propension au masochisme. ${ }^{14}$

Si la définition partipriste de l' «autre» ne coöncide pas tout à fait avec celle de Lapointe (j'y reviendrai plus loin), l'idée de transformer l'instinct de vie en instinct de mort (et par conséquent d'agir sur cette transformation pour rétablir l'instinct de vie) traverse tout le poème en question: l'homme est petit, pauvre, humilie, futile, soumis au règne de la terreur (qui vrombit comme un dieu, p. 209), les images de mort lui collent à la peau... Toute la première partie du poème situe l'homme dans la négativité.

Survient alors un événement au sens diégétique du terme qui fait basculer l'imagerie du poème dans la positivité: la grève. L'événement joue un rôle crucial parce qu'il mobilise les forces disséminées du groupe des travailleurs et coïncide avec le début de la révolte appelée par le titre. Si l'on s'en tient encore au contenu du poème, il est facile de repérer une série d'oppositions quasi symétriques entre la première et la seconde partie qui témoignent de son euphorisation grandissante.

À part quelques exceptions déjà signalées à propos de l'inversion rhétorique, la première partie du poème donne à la durée, au temps qui passe, une valeur toute négative: linéaire, irréversible, il ne produit jamais que du futile, du déjà mort. À mesure que l'écoulement des fleuves dépossède l'homme en le dépassant, l'humain s'échappe de l'homme. Le temps oppressif soumet ce demier à l'absurdité d'un travail qu'il doit recommencer jour après jour comme les danaïdes occupées à remplir un tonneau sans fond:

danaides

les fleuves coulent

avec eux les villages (p. 209).

14 André-J. Bélanger, op. cit., p. 146. 
Si l'on se reporte à la deuxième partie du poème, on retrouve le sème du temps, mais cette fois inversé dans le sens d'une valorisation: les années sont volontaires (p. 211), nous sommes portés par l'eau (p. 212). Le mouvement du sujet et celui du temps sont synchronisés. L'eau devient navigable, le temps qui passe prend la mesure de l'homme. Autre glissement significatif: tandis que le présent domine dans les premiers verbes, le futur (confirmé par les termes «demain», «espoir», «prophétique») prend le dessus à la fin. La perception du temps est renversée tête-bêche: au temps statique mais fuyant du début s'oppose le temps élastique, le temps du mouvement assumé; on se permet de regarder loin en avant, on se donne l'avenir à penser, ce qui était refusé au travailleur prisonnier du rythme obsédant et vide de la répétition.

Filons une autre image récurrente dans le poème: celle de la fécondité. J'ai écrit plus haut que «la mise à mort» en début de poème s'avoue inefficace, absurde, irrécupérable pour la cause révolutionnaire. Si mourir ne sert à rien, naître non plus (maternités futiles). «Fertile» et «futile» sont les deux épithètes charnières du renversement de l'éthos poétique. L'intolérable, le tragique dans ce poème, ce n'est pas la mort, ni même la peur mal définie qui accable le «petit homme»: c'est la futilité (de la mort, du travail, de l'enfantement). L'adjectif «futile» apparaît à trois moments dans la première partie, sans compter les termes dont les sèmes s'y rattachent (vides, vaines, illusoires). Or l'image maternelle s'inverse complètement dans la seconde partie où la fécondité est associée à des sensations de chaleur (filles de chaleur) et à la jubilation des corps (baiser, caresses). Les derniers verbes (savent posséder) sont l'exact envers positif du verbe initial (nul $n^{\prime} a$ ). Le demier distique, dont le ton aphoristique rappelle d'ailleurs l'ouverture, célèbre la possession physique du corps de la mère:

les fruits savent posséder l'âme comme l'étalon la mère (p. 212).

Plus que la terre et les fleuves, c'est le corps qui est l'enjeu de la révolte. Encore faudrait-il dire l'instinct génésique du corps, la capacité et la volonté de faire d'autres corps, de donner vie. «Fruits» est le symbole par excellence de la fécondité et c'est sur l'image de la reproduction que s'achève le poème.

Il est frappant de constater, à la lumière de cette première lecture, à quel point le poème se présente sous la forme d'un renversement symétrique. Dans l'ordre de l'imaginaire archétypologique, le contraste entre les deux parties est extrêmement appuyé. Alors que la première moitié du poème est placée sur l'axe horizontal du terre à terre, du temps linéaire, l'autre moitié se situe manifestement dans la verticalité. Une strophe en particulier illustre la fortune de ce que Gilbert Durand appelle les symboles ascensionnels:

demain s'amoncelle dans l' altitude des caresses dans le nid du délire s' envolent des oiseaux multiples des villes profondes et légères des forêts de liqueurs une planète fervente innombrable (p. 212). 
Les termes «amoncelle», «altitude», «nid», «s'envolent», «oiseaux», «villes», «légères» et «forêts» comportent tous le sème de la verticalité. Dans la littérature mythologique ou religieuse, ces termes ascensionnels se rapporteraient à la conquête d'un idéal (en général l'immortalité). Durand écrit que les symboles ascensionnels nous apparaissent tous marqués par le souci de la reconquête d’ une puissance perdue, d' un tonus dégradé par la chute ${ }^{15}$. L'élévation dans le poème constitue bel et bien une reconquête, une réappropriation d'un espace dont on avait perdu la possession. L'aspiration à une certaine hauteur, à un absolu, s'exprime par les caresses, par un geste du corps (plutôt que de l'esprit). Lapointe accorde au corps une valeur et un pouvoir ascensionnels.

Enfin, illustrons le renversement de la manière la plus simple, en prélevant le même mot à deux endroits différents. Si la mise à mort du début est négative, ce n'est plus du tout certain au moment où le dernier homme sera fusillé d' une tendresse / mortelle (p. 211). Être fusillé par la tendresse, même mortelle, est-ce encore mourir? Il s'agit dans ce cas-ci d'une mise à vie, d'une renaissance, et l'épithète «mortelle», placée derrière le substantif «tendresse», n'a pas de sens négatif: «mortelle» doit se lire par opposition à ce qui est immortel, c'est-à-dire à ce qui n'est pas de caractère humain. Toute allusion à la futilité de la mort est disparue; celle-ci est au contraire enregistrée dans le livre de misère et c'est cette trace écrite, cette mémoire indélébile qui assure la vie humaine: la misère s'accroûtra d' une feuille perverse I animale où s' allumeront les artifices du cri et du sommeil (p. 211). Le geste de mourir ne prend son sens que s'il est fécondé par le geste d'écrire. Si l'on est passé du chaos à ce qu'on appellera l'utopie (le non-lieu de la tendresse), c'est donc avec le concours de la médiation langagière. Par ailleurs, entre les saisons champêtres (p. 208) et les humaines saisons / savoureuses saisons (p. 211), la connotation du mot «saison» a change de signe. Le bucolisme de «champêtres» fait écho aux promenades aux bois le dimanche (quelques lignes plus haut). Le jour de repos ou la semaine de repos durant la saison estivale font partie de la même grille horaire à laquelle est soumis le travailleur. Le dimanche, la semaine de vacances, l'heure des repas (repas nécessaires, p. 208) et le souvenir des morts (inoubliables morts, p. 208) sont tout ce qui lui reste de vivant. On remarque en outre que l'ordre du syntagme dans lequel apparaît le mot «saisons» s'inverse dans la deuxième partie, ce qui déplace l'accent sur les épithètes «humaines» et «savoureuses». Le dernier exemple que je relèverai est celui de la ville. Dans la première moitié, les villes abritent des pauvres, elles sont renfrognées (p. 208), molles (p. 209), sécrètent des cristaux opaques / missiles de chaque jour (p. 209) et la terreur y vrombit comme un dieu (p. 209). Or la seule occurrence du mot «ville» dans la seconde partie est celleci: des villes profondes et légères (p. 212), vers qui s'inscrit dans la strophe extatique décrite plus haut.

15 Gilbert Durand, Structures anthropologiques de l'imaginaire. Introduction a l'archétypologie générale, Paris, Presses universitaires de France, 1960, p. 148. 


\section{Des idéologies en colloque}

Un certain nombre de valeurs accompagnent l'idéologie de la révolte, qu'il serait intéressant de comparer avec celles que véhicule Parti pris. J'ai affirmé que la «révolte de terre» visait le capitalisme. Le côté inhumain du capitalisme proviendrait essentiellement du fatalisme entropique qui paralyse les masses et fait le jeu des dirigeants. L'effet de l'industrialisation à l'américaine est de mécaniser les gestes des travailleurs. Toutes les images paniques du temps qui fuit expriment avec urgence ce déterminisme qui rend l'homme inerte.

Au contraire, le discours prophétique (possession prophétique, p. 212) qu'on lit dans la seconde moitié du poème insiste à plusieurs reprises sur le caractère volontaire et humain des contingences spatio-temporelles. Après avoir parlé des «artifices» du cri et du sommeil, Lapointe résume dans une strophe cette émergence d'une volonté humaine:

et le délire que nous avons connu

d' alimenter l' espace années volontaires ô survivantes

nous le tenons bouche a bouche

comme un cri (p. 211).

Nourrir l'espace et le temps comme s'ils étaient entièrement produits par l'homme, c'est en prendre possession. Le désir conscient, volontaire, constitue dans le poème une valeur humaine primordiale à partir de laquelle se manifeste l'audace de posséder la terre (p. 211). On reconnaît là un des présupposés de la position partipriste qui fonde son analyse, explique Bélanger, sur la conscience comme révélateur d'un état social et aussi comme moteur de l'action 16 . Pour Parti pris, il ne fait pas de doute que la révolution n'aura lieu que si elle est appuyée par une intelligentsia révolutionnaire: au stade où en est Parti pris dans ses rapports avec les classes laborieuses, il est presque entendu que le gros du travail revient à l'intelligentsia qui devra affranchir les consciences encore mystifiées $^{17}$.

À ce titre, les poètes sont invités à jouer un rôle majeur. La question n'est pas de savoir si le poète a, oui ou non, rempli son rôle; il s'agit plutôt de tirer toutes les conséquences de la confiance que le penseur révolutionnaire place dans le poète. Pour Parti pris, la nouvelle culture québécoise se définit par opposition à tout ce qui relève du capitalisme, mais appartient tout comme le poème à l'ordre de la prophétie. Bélanger n'hésite pas à associer les visées révolutionnaires de la revue à la récupération d' une harmonie perdue ${ }^{18}$. Entre le discours tenu à la rigueur des faits et le discours entraîné par l'idéal projeté, le choix est clair: la dimension prophétique de la parole est attestée plusieurs fois par Parti pris. Pierre Maheu ócrit par exemple: Nous n' avons qu' une chose a faire: dire et

16 André-J. Bélanger, op. cì., p. 145.

17 Ibid., p. 179.

18 Ibid., p. 162. 
appeler la révolution jusqu'à la susciter enfin; nous disons, nous écrivons des choses qui ne sont pas tout d fait vraies pour qu' elles le deviennent ${ }^{19}$. C'est en ce sens que le discours prophétique participe au processus révolutionnaire. De son côté, Paul Chamberland écrit ceci qui pourrait bien servir d'épigraphe au poème de Lapointe: Nous nous exilons, mais dans l'avenir ${ }^{20}$. Pour beaucoup, la Révolution tranquille a d'abord été une prise de parole par différents groupes sociaux jusque-là tenus à l'écart des débats publics. Ce pouvoir de la parole se manifeste aussi dans le poème de Lapointe, tant implicitement qu'explicitement. Ainsi le refrain $\hat{o}$ psalmodie $\hat{o}$ psaumes est une formule incantatoire, magique. En la prononçant onze fois, le lecteur opère un charme, il transforme le déjà-là en un futur, la parole agit, au sens des pragmaticiens. Le langage, autant chez Lapointe que chez Parti pris, a une valeur révolutionnaire.

Parti pris est, comme le poème de Lapointe, une fervente prise de parole par laquelle l'invention est toujours à portée de discours: changer les Canadiens français en Québécois dans les mots, c'est se rapprocher d'une réalité québécoise. Ainsi le discours partipriste et le poème aspirent tous deux à transformer le monde en s'appuyant sur la parole, en appelant à l'existence cette nouvelle société qu'ils souhaitent.

À l'origine de cette prise de parole, il y a la conviction que l'acte et la parole ne se contredisent pas, que parler c'est agir. On se souvient que Breton s'était élevé dans le premier manifeste contre la scandaleuse opposition entre le rêve et la réalité. Pris dans ses contradictions entre la révolution hors et dans la littérature, le surréalisme s'est finalement sabordé comme mouvement lorsqu'il s'est rangé du côté de l'esthétique ${ }^{21}$. Parti pris a souffert tout au long de sa brève existence de la même hésitation entre le dire et le faire. À bien des égards, il semble que ce soit le discours poétique qui ait contaminé le discours social de la revue, et non l'inverse. Celui qui écrit dans Parti pris a généralement la conscience malheureuse de l'écrivain déchiré entre l'écriture et la non-écriture, c'est-àdire entre un discours taxé d'irresponsabilité, un discours qui n' exprimerait, si je cédais à ses charmes, écrit Hubert Aquin, que la domination dont je suis le lieu depuis deux siècles ${ }^{22}$, et un discours engageant qui agit directement sur la réalité quotidienne. C'est dans cet esprit par exemple que Maheu se sent tenu de justifier la décision de consacrer un numéro spécial à la littérature québécoise:

Nous avons écrit récemment que la révolution était un travail sérieux et qu'il fallait commencer de la faire au lieu de la rêver, nous avons annoncé que la revue serait consacrée da l'analyse des problèmes sociaux

19 Pierre Maheu, «Que faire? », Partl pris, vol. I, $n^{\circ} 5$, février 1964, p. 44-45.

20 Paul Chamberland, «Nous avons choisi la révolution», Parti pris, vol. I, n ${ }^{\circ}$, février 1964 , p. 3.

21 C'est à ce signe, à savoir la reconnaissance définitive d'une esthétique, que Maurice Nadeau reconnant le moment de la faillite du mouvement surréaliste : $C^{\prime}$ est en somme d partir de ce moment, d partir du moment où Breton se range, qu'il le veuille ou non, dans la catégorie des Artistes, que nous daterions l'avortement du mouvement surréaliste. (Histolre du surréalisme, Paris, Seuil, 1945, p. 234).

22 Hubert Aquin, «Professsion: écrivain», Parti pris, vol. I, n 4, janvier 1964, p. 26. 


\section{et politiques, et nous voild en plein manifeste littéraire, est-ce bien sérieux? 23}

Plusieurs membres de la rédaction sont des littéraires (Chamberland, Brochu, et dans une moindre mesure Maheu), au risque de se voir confiner comme les surréalistes au rôle de «phraseurs révolutionnaires». Parler, pour Parti pris, fait problème. On parle pour agir, non plus pour parler, mais aussi un peu faute d'agir. En 1965, Aquin lance: écrire me tue ${ }^{24}$. En même temps qu'elle est prophétique, la parole partipriste se sent toujours un peu coupable, ambitieuse, provocante mais assaillie par ailleurs de doutes sur son efficacité à résoudre les contradictions de la société qu'elle dénonce. S'il est assez inconfortable de parler pour Parti pris, d'être donc à la fois en puissance et en faute d'agir, le poème jouit en revanche d'une tribune qui lui évite de douter de sa légitimité et qui permet à la parole de se déployer avec une liberté, une autonomie et une force qui sont refusées au discours d'une revue. Le poème affiche en effet, comme on le verra dans la suite de cet article, une sorte de soucieuse insouciance devant les conditions d'existence qu'il réprouve.

Le rêve d'une réconciliation humaine est modalisé dans le poème sous la forme d'une appropriation de la terre. Ce thème apparaît dans plusieurs des premières œuvres publiées aux éditions Parti pris et Robert Major l'interprète ainsi: Cette thématique de la repossession (de soi, d' un espace) est l'incarnation réussie d' une idéologie de la décolonisation 25.

On sait que Parti pris considérait que l'aliénation des Québécois était due à ce qu'ils étaient colonisés et exploités aux niveaux politique, économique et culturel. Lecteurs de Fanon, Memmi et Berque, les partipristes faisaient de la décolonisation un préalable à la révolution socialiste. On se gardera cependant de lire directement cette «idéologie de la décolonisation» dans le poème de Lapointe. L'identification de la cause québécoise à la cause algérienne constituait du reste une stratégie douteuse pour dramatiser les problèmes domestiques en les auréolant du prestige de la guerre d'indépendance algérienne. En ce qui concerne le poème de Lapointe, la repossession dont parle Major s'interprète difficilement en fonction de la décolonisation. Elle correspond véritablement à une recrudescence des sensations (tendresse, parfum, caresses, savoureuses, chaleur, plaisir) et coïncide avec le réveil, après hibernation (dieu que l' hiver est dur mais précède mai, p. 210), du corps.

J'ai déjà souligné que le signe définitif de la possession réussie est le corps: nous le tenons bouche d bouche / comme un cri (p. 211), tout se trame dans un baiser (p. 211), mère des plaisirs (p. 212). La possession, terme clef du poème, se lit sur plusieurs registres (économique, politique, magique...) mais la primauté du registre érotique ne fait pas de doute. Que signifie cette valorisation du

23 Pierre Maheu, «Le poète et le permanent», Parti pris, vol. II, $\mathrm{n}^{\circ}$ 5, janvier 1965, p. 2.

24 Hubert Aquin, loc. cit., p. 23.

25 Robert Major, Parti pris. Idéologies et littérature, Montréal, Cahiers du Québec/ Hurtubise HMH, coll. \&Littérature», 1979, p. 258. 
corps dans le cadre du projet initial de révolte? Que la caresse soit le geste même de la révolte, et non pas la pierre ou le feu du départ, voilà qui témoigne peut-être du «pacifisme» de l'auteur ${ }^{26}$. Mais l'érotisme de la poésie de Lapointe m'intéresse surtout dans la mesure où il conduit à une dimension essentielle du «réel absolu».

Le corps a quelque chose d'absolu chez Lapointe. Chamberland écrivait en 1965: la sensualité presque sacrée marque tout le déploiement de la poésie de Lapointe. [...] Sentir est immédiatement naître et fonder ${ }^{27}$. Décrire l'homme, c'est souvent chez Lapointe décrire un corps humain: les muscles, la sueur, le cœur, les reins, les os, la chair, l'artère, les côtes. La perception de l'homme en situation est tout entière inscrite dans la perception de l'espace qu'occupe le corps: plus que tout autre qualificatif, l'épithète «petit» détermine l'homme dans sa globalité. Aussi, poursuit Chamberland a propos de la poésie de Lapointe, plus que la vue, le tact y est la sensation première, fondatrice: proximité, contact, caresse et pénétration. ${ }^{28}$ Il est significatif que Lapointe ait choisi de nommer ceux qui n'appartiennent pas à la classe des travailleurs «les sublimes»:

les sublimes s'entourent coiffés de plumages
d' anneaux (p. 209).

Qui sont ces sublimes? Hommes «hauts» par opposition à «petit homme» (le pluriel s'oppose aussi au singulier), «les sublimes» n'ont pas de corps: leurs plumages et leurs anneaux sont des signes de l'extérieur qui forment un écran de beauté sans profondeur, à deux dimensions. Par écran, il faut surtout entendre masque, illusion; les plumages et les anneaux évoquent un déguisement, et renvoient plus précisément à une parade où les sublimes imitent de manière superficielle et risible les pratiques des autochtones: ils se réunissent en cercle et portent des plumes et des anneaux. L'imitation est ici avant tout dérision, non pas des rites des tribus autochtones mais de l'attitude fausse des sublimes. Le choix de ce terme signale d'ailleurs l'ironie du propos: êtres sublimes ou êtres sublimés, c'est-à-dire dont l'énergie des pulsions sexuelles est transformée au profit d'une énergie socialement acceptable. En tant que sujets sociaux, les sublimes se situent à un rang élevé dans l'échelle sociale; la prononciation même du mot avec ses deux voyelles pointues évoque le raffinement, voire la préciosité de la bourgeoisie. En tant que sujets poétiques enfin, les sublimes ont une fonction équivalente à celle d'une pièce de décoration, ce sont les dentelles de la société et ils ne participent d'aucune façon (sinon comme opposants justement) à

26 Cette interprétation prête le flanc au réductionnisme mais s'appuie sur une quantité impressionnante de mots qui s'indexent sur une isotopie /militaire/: blesses, mise à mort, sang, feu, aigle, cavalier, proie, rompt, missile, armes, paix guerrière, etc.

27 Paul Chamberland, «Fondation du territoire», Partl pris, vol. IV, nos 9-10-11-12, maisoût 1967, p. 21.

28 Ibid., p. 36. 
la révolte en cours ${ }^{29}$. Seul le corps est révolutionnaire, le corps pour le cour et pour les âmes, mais le corps d'abord.

La révolution sexuelle a banalisé jusqu'à un certain point cette volonté de posséder son corps, de douer la matière de désirs, d'animer tout le réel d'une érotique, de faire que les objets se convoitent / les uns les autres («Gravitations», p. 214). Mais cette appropriation du corps dépasse les simples questions d'hygiène physique et donne sur autre chose que le mieux-être et la satisfaction. Le bien, l'espoir et la beauté sont des mots vides: si le dieu qu' apostrophent les ors n' allait être que charme (p. 212), lance avec inquiétude le poète. La beauté seule (les ors) ne constitue jamais une valeur dans ce poème: pour être favorable, la beauté doit être féconde. C'est en possédant la matière (la terre) que la beauté du «petit homme» devient autre chose que de la pure beauté. Cette opposition entre l'intérieur et l'extérieur, entre l'appropriation superficielle et illusoire et l'investissement au plus profond du corps est manifeste dans tout le poème: les arêtes des mondes sont illusoires (p. 210) s'oppose à depuis, elle tourne au vent qui sourd de l'intérieur (p. 210) et à la profondeur des côtes (p. 211). Posséder, c'est mettre à l'intérieur de soi, c'est accepter de se laisser envahir par ce à quoi on tient, c'est donc aussi être possédé: nous sommes portés par l' eau et le délire (p. 212).

La sensation chez Lapointe a quelque chose de solidaire: c'est se permettre à soi, en même temps qu'aux autres, d'entrer en contact le plus intimement possible avec l'extérieur. Pour Lapointe, est sociale toute poésie assumée charnellement ${ }^{30}$. Le social passe par le chamel, la révolution socialiste est indissolublement liée, comme chez les surréalistes, à la révolution sexuelle. L'intime libéré, le privé assumé, le contact privilégié de l'amour, tout cela ordonne d'une certaine manière la transformation de la société capitaliste. Il n'y a donc pas d'opposition entre l'individuel et le collectif. Le «petit homme» décrit par Lapointe est irremplaçable et multiple, différent et innombrable. Le poème renonce à faire du seul individu le sujet efficace de la révolte mais il n'abolit pas pour autant la singularité de l'homme.

Le rapport de l'individuel au collectif implique des présupposés similaires chez Parti pris. La revue dénonce l'individualisme en tant que produit du libéralisme bourgeois. Dans sa critique du système démocratique, Maheu accuse la classe possédante de diviser la classe des travailleurs et d'empêcher du même coup une prise de conscience collective. Dans cette perspective, explique Bélanger, l'homme affirme sa liberté dans sa séparation d'avec ses semblables. Fondée sur le chacun pour soi, la libre concurrence etc., cette conception de l'aménagement social projette le modèle d'un collectif divisé, atomisé et parcellisé. ${ }^{31} \mathrm{La}$ volonté de faire l'unité de la classe des travailleurs constitue un

29 Les sublimes, opposants au même titre que les patrons, sont traités sur le même mode ironique. Le poème emploie pour parler de ces oppositions un discours de la distance qui annule le pathos propre à ces conflits.

30 Paul-Marie Lapointe, «Poésie sociale et morale», op. cit., p. 87.

31 André-J. Bélanger, op. cit., p. 170. 
des enjeux primordiaux du programme social de Parti pris. La révolution partipriste vise en définitive à rassembler les individus dans une conscience commune fondée sur l'idée d'appartenance et sur une sorte de camaraderie qui amènerait un libre mouvement de reconnaissance entre chacun. Dans ce contexte, il est assez paradoxal de lire chez Bélanger que Parti pris propose une stratégie globale mais avec un dispositif plutôt accordé d l'action individuelle ${ }^{32}$. Les formules oxymoriques telles que le «moi collectif» ou le «nous intersubjectif» définissent ce nouveau rapport que l'individu doit entretenir avec la société. Désormais l'action de chacun résonne dans la collectivité, désormais la parole de l'un, parce qu'elle est vraie pour l'un, est aussi responsable pour l'autre. Bélanger reprend la pensée de Chamberland sur ce thème:

\section{Ma liberté est donc liée à la libération commune, le «je» et le «nous» se trouvent engagés dans un même élan, qui fera éclater «ce vrai qui est nous-mêmes, en assumant le particularisme et l'exceptionnel qui nous séparent des autres». Au-delà d'une identité nationale réintégrée, il y a recherche d' un homme québécois réintégré lui aussi d̀ une collectivité réelle. ${ }^{33}$}

Que la dimension sociale de la poésie de Lapointe passe par l'expérience individuelle ne surprend donc guère le lecteur de Parti pris: l'individu, à ses yeux, est partie prenante au combat de la société, le privé, au prix de rapides médiations, conduit au social.

«Psaume pour une révolte de terre» partage avec le discours partipriste plusieurs éléments de sens: révolte contre le capitalisme, célébration du quotidien, parti pris pour le travailleur, appropriation d'un espace, avenir prophétique, puissance de la parole. Mais les questions du sujet et du psaume vont accentuer désormais les différences entre les deux discours, sans pour autant rendre caduque la première lecture plus générale proposée jusqu'ici. Il ne s'agit donc pas d'élaborer un réseau de significations parallèles à celui qui a déjà été dégagé, mais d'éprouver et de modifier le cas échéant cette lecture.

\section{Du pareil à l'autre}

La thématique de la fondation du territoire, analysée par Chamberland dans un article écrit en $1967^{34}$, sert d'après lui de toile de fond à toute une séquence de la poésie québécoise dont Lapointe fait partie. Mais si le territoire est forcément découpé d'après les frontières nationales dans le discours de Parti pris, il déborde largement ces frontières dans le discours des poètes, et la poésie du pays ne constitue, selon Chamberland, qu'une variante de la thématique de la fondation. À l'opposition reconnue par le nationalisme partipriste entre l'ici et l'ailleurs, on préfere la position d'un ailleurs qui ne se sent jamais tenu de se situer par rapport à un quelconque centre. Le corps excentrique, extatique, est le lieu, ou plutôt le

32 Ibid., p. 156.

33 Ibid., p. 162.

34 Paul Chamberland, «Fondation du territoire», loc. cit., p. 11-42. 
non-lieu de la révolte. On parlera, dans l'esprit de Philippe Lacoue-Labarthe, de l'achèvement utopique de la révolte:

Et de fait la volte ou la révolte de l'appropriation n'a pas lieu. L' ricimaintenant " de l'existence singulière est immédiatement un ailleurs et un autre temps. Si l'appropriation advient, c'est dans l'u-topie même, on le sait. C'est pourquoi, au partage topologique de l'ici et de l'étranger, du proche et du lointain, qui assigne - quoi qu' on essaie de dire - des places, il est nécessaire de substituer le partage, non localisable, de la différence ou de l'altérité. Au lieu (sans lieu) de l'ailleurs survient alors un kautre», c'est-d-dire un existant singulier au nom de qui - et cette fois, l'expression est juste - le poème entretient l'espoir de parler. 35

La notion d'altérité permet d'atteindre quelque chose de central dans la poésie moderne. L'étrange, le dissonant, le paradoxal sont tous des tentatives extrêmes pour confronter le réel ordinaire à un «autre» réel. Cette notion est convoquée jusque dans l'analyse des opérateurs thétoriques du poème. De manière succincte, il est possible de montrer que les transformations rhétoriques les plus marquantes de «Psaume pour une révolte de terre» misent sur la primauté de l'autre dans son opposition au même.

Sans aller jusqu'à quantifier l'emploi des tropes, il semble que Lapointe, dans ce poème, préfere entre tous l'oxymore. Cela n'empêche pas la synecdoque particularisante ou la métonymie de jouer un rôle important, mais moins que l'oxymore. Au tournant du poème, c'est-à-dire littéralement au moment où le vent tourne (depuis, elle tourne au vent qui sourd de l' intérieur, p. 210), Lapointe déploie une telle série d'expressions antonymiques qu'on ne peut s'empêcher d'associer le procédé métorique à l'effet jubilatoire qui se dégage de ce passage. On trouve entre autres la forme la plus avérée de l'oxymore dans la contradiction entre deux mots voisins, l'un substantif, l'autre adjectif: paix guerrière, guerre pacifiante, tendresse mortelle. Les autres oxymores, de forme moins classique, sont: animal/artifices (nature/culture), lins du volcan (douceur/violence) et ostensoirs de caresses (sacre/profane). S'il faut s'abstenir d'accorder plus de sens qu'elles n'en ont aux articulations rhétoriques, nous retiendrons tout de même ce qu'en dit le groupe MU:

A cet égard, parmi les tropes servant à la médiation, la métaphore s'impose comme centrale, comme la figure ureine». C'est que, dans l'analogie, elle maintient en équilibre les droits du Même et de l'Autre, alors que, d' un côté, la synecdoque n'assimile guère que le Même au Même et que, de l'autre, la métonymie ou l'oxymore «forcent», chacun d sa manière, l' analogie, en la faisant reposer sur des différences. ${ }^{36}$

35 Philippe Lacoue-Labarthe, De la poésie comme expérience, Christian Bourgois éditeur, coll. «Détroits», 1986, p. 90.

36 Groupe MU, Rhétorique de la poésie, op. cit., p. 210. 
Contrairement à l'antithèse, où les deux termes antonymiques ne sont pas en relation syntaxique, l'oxymore opère ce que l'on a appelé la coïncidentia oppositorum. La distinction entre l'antithèse et l'oxymore nous apprend encore, sur le plan de leur éthos respectif, que la contradiction [est] tragiquement proclamée par l'antithèse, paradisiaquement assumée par l' oxymore. ${ }^{37}$

Il semble que la béance devant l'autre, le trou du poème, soit comblé par ce qui est précisément impossible dans le discours de Parti pris, comme dans tout discours idéologique: le paradoxe affiché en tant que tel, en tant que possible suspendu, maintenu, résistant farouchement à la tentation de se résoudre comme le voudrait le modèle dialectique. L'altérité est a priori associée au paradoxe parce qu'elle est impensable sans son rapport d'opposition au même. Entre le même et l'autre, écrit Lacoue-Labarthe, il y a nécessairement rapport, rapport réciproque ou plutôt, comme disait Hölderlin, échange. ${ }^{38}$ Mais malgré leur caractère indispensable l'un à l'autre, les deux termes de cette opposition sont d'inégale force: l'altérité l'emporte puisqu'elle sous-tend le mouvement impliqué par leur mise en rapport: dans le «se rapporter d», c'est par définition le mouvement de l'altération qui prédomine. ${ }^{39}$

J'ai écrit plus haut que la réappropriation du corps revendiquée par le poème en tant qu'épreuve libératrice suggère un contact intime entre l'homme et l'extérieur. Le privilège accordé à la sensation ne va pas sans une certaine vulnérabilité du corps, désormais menacé par l'impropre. Le désir d'un corps adverse implique l'assomption d'une altérité permanente. Ce n'est pas seulement le schéma dialectique de Hegel qui est récusé par là, c'est toute sa notion de conscience de soi unifiée ou achevéét ${ }^{40}$. L'épreuve du corps est donc aussi une expérience au sens fort, étymologiquement l'épreuve d'un danger.

Le «nous» partipriste est un «nous-nation» qui revendique le nom de Québécois plutôt que celui de Canadien français et qui s'inspire encore beaucoup, d'après Bélanger, du nationalisme groulxiste. L'identité nationale brisée par la Conquête constitue la base de la réflexion aussi bien de Groulx que de Parti pris. Cependant les membres de la revue rejettent la composante catholique de cette identité, ce qui les oblige à consolider le portrait du Québécois en le situant par rapport à ce qu'il n'est pas et qui constitue une menace pour son intégrité: Parti pris, écrit Bélanger, mise amplement sur le duel entre francophones et anglophones, le nous québécois aux prises avec cet autre «Canadian» dont la présence

37 Groupe MU, Rhétorique générale, Paris, Librairie Larousse, coll. «Langue et langage», p. 120.

38 Philippe Lacoue-Labarthe, De la poésie comme expérience, op. cit., p. 90-91.

39 Ibid., p. 91.

40 Julia Kristeva a critiqué d'un point de vue psychanalytique la notion du désir chez Hegel: Notons la marque aparanoïaques dans ce parcours du désir : la conscience de soi se constitue par la suppression de l'Autre hétérogène, et le désir est cette suppression même; depuis toujours sur la lancée du désir, la econscience de soi» devient son Autre sans s'abandonner pour autant comme telle (la Révolution du langage poétique, Paris, Seuil, coll. «Points», 1974, p. 123). Le nous partipriste correspond en ce sens à la conscience de soi hégélienne. 
est négation de la nôtre. ${ }^{41}$ Bélanger va même jusqu'à accorder une fonction cathartique au joual et au mouvement Ti-Pop qui permettent au «nous» de se libérer de son aliénation. La révolution pour Parti pris ne s'arrête pas à la constitution d'un «nous authentique», mais l'accent est là: le nationalisme, écrit Chamberland, est le seul mode de conscience homogène, et totalisant de la société québécoise. 42

La solidarité ethnique et culturelle de Parti pris ne correspond pas au «nous» présent dans le poème de Lapointe. L'emploi de ce pronom y est rare et particulier. L'instance élocutoire, presque absente dans l'ensemble du poème, affleure régulièrement dans les parenthèses: que ferons-nous (p. 207), mes villes (p. 208), mes agités (p. 208), nos os (p. 210), nous les enlaçons (p. 211). En fait, le régime impersonnel domine partout dans le poème sauf dans les parenthèses où le sujet (individuel ou collectif) est discrètement restitué. La personne n'existe qu'en arrière-plan, en voix off, dans un murmure distant qui perturbe à peine l'ordre de l'énonciation objective. Dans (que ferons-nous de cette pierre et de ce feu?), le pronom «nous» ne renvoie à personne, il est tout entier porté par une question, dont il n'est d'ailleurs pas l'objet. Le «nous» advient comme s'il était connu du lecteur (comme les deux démonstratifs «ce», qui suggèrent le connu, le familier), mais son existence lui échappe, doublement conditionnelle: d'une part, il doit émerger du sous-sol de l'interrogation et de la parenthèse, d'autre part il surgit en fonction de son destin, sa présence est littéralement «à venir» (c'est le seul verbe qui soit au futur dans la première partie du poème).

Il est frappant que la dernière occurrence du pronom «nous» (seigneur que deviendrons-nous?) s'écrive aussi sous la forme interrogative et au futur. Même si ce pronom est à deux occasions à l'avancée du poème (nous la voulons, p. 210, nous le tenons bouche a bouche, p. 211), son utilisation n'est pas appuyée et l'identité qu'il recouvre semble moins se référer à une collectivité présente que future. Le surgissement de l'énoncé nous la voulons en conclusion de la plus longue séquence du poème a ici valeur d'embrayeur générique parce qu'il entraîne le poème sur un autre registre temporel qui est celui du projet, du futur indéterminé, de la prophétie.

Le tracé idéologique de Parti pris se résume essentiellement à «la recherche d'un collectif» (c'est le titre du chapitre que Bélanger lui consacre) qui serait basé sur une identité nationale. Le brevet collectif semble n'être accordé qu'avec la suppression de l'hétérogénéité des êtres qui constituent la nation. L'unité se trouve constamment menacée par un mouvement de scission dû à l'autre. C'est sur ce fond d'homogénéité très appuyée que le fragmentaire et le paradoxal (tout le registre religieux du poème notamment) prennent toute leur valeur. Il s'agit d'une différence idéologique capitale entre les deux types de discours: tandis que Parti pris unifie sans cesse ses discours, les fait converger vers l'identité nationale et la révolution socialiste, «Psaume pour une révolte de terre» entretient

41 André-J. Bélanger, op. cit., p. 155.

42 Paul Chamberland, «Les contradictions de la révolution tranquille», Partl pris, vol. I, $n^{\circ}$ 5, février 1964, p. 13. 
plusieurs discours à la fois sans jamais les réduire à une seule et même instance, qu'elle soit subjective ou collective.

Le «nous» de Lapointe semble dessiner les contours d'une classe sociale plus que d'une classe ethnique. Il comprend les travailleurs, les petits, les exploités du système. Les peuples humiliés petits du monde (p. 210) ont fort peu à voir avec le collectif désigné par Parti pris. Le terme «travailleur» dans le vocabulaire partipriste regroupe tous les salariés qui vendent leur travail au profit d'un employeur, c'est-à-dire les cols blancs, les travailleurs ruraux, les cultivateurs et surtout les intellectuels. En théorie, Parti pris est aussi de tendance socialiste, mais dans les faits, conclut Bélanger, Parti pris s'applique à désigner le nous exprimé par le milieu de l'enseignement (enseignants et enseignés), le cinéma, la télévision, la presse, etc. ${ }^{43}$ En dernière analyse, ce socialisme est subordonné aux impératifs de la révolution nationale: Le socialisme de Parti pris s'insère dans l'économie révolutionnaire comme préoccupation seconde sans être pour autant négligeable. Il sert presque toujours à qualifier la révolution nationale qui sans lui se trouverait dépourvue de contenu. ${ }^{44}$ Le socialisme inscrit dans le poème, s'il n'est pas moins rudimentaire que celui de la revue, ne s'articule pas autour d'une idéologie de l'identité nationale. La revalorisation de soi ne passe pas par un renfermement, fut-il temporaire, autour d'une identité unifiée, mais par une ouverture vers l'autre, par l'éloge de la multiplicité, de l'incomplet, de l'échange.

La voix dans la parenthèse joue dans le poème un rôle particulier qui n'est pas étranger à ce morcellement du sujet. Sont supprimés dans «Psaume pour une révolte de terre» tous les éléments de ponctuation, sauf deux virgules, deux tirets, quatre points d'interrogation et sept parenthèses. Les trois premiers signes se situent souvent à l'intérieur des parenthèses, ce qui confere à ces dernières un certain prestige dans l'économie du poème. Comme elles ne revêtent pas le caractère habituellement explicatif des parenthèses dans le discours fonctionnel, elles recèlent une certaine dose d'étrangeté. Et même si elles ne rompent pas toujours avec ce qui précède et suit, les mots qu'elles contiennent sont des apartés. On cherche en vain une fonction récurrente de la parenthèse. Dans l'impulsion fragmentée du poème, elle en accentue certainement l'aspect parataxique mais son rôle ne s'arrête pas là. Par la fréquence du procédé, le lecteur est attentif, malgré la discontinuité apparente, à l'enchaînement de ces énoncés placés dans un creux textuel qui les isole du reste du poème. Les parenthèses forment ainsi une configuration paradoxale: marginales par rapport au reste du poème, elles deviennent du fait de leur abondance éminemment visibles et signifiantes. Pour mieux percevoir le sens qui s'en dégage, les voici réunies bout à bout:

43 André-J. Bélanger, op. cit., p. 188.

44 Ibid., p. 173. 
1. (que ferons-nous de cette pierre et de ce feu?)

2. (mes villes aux pauvres pauvres dans l'aisselle des coffres mes agités

futiles

$$
\text { travailleurs) }
$$

3. (musaraignes de la mort

$$
\text { hommes femmes enfants) }
$$

4. (aciers de couleurs aluminium titanes impassibles alliages nos os futiles reposent dans leurs chairs)

5. (depuis, elle tourne au vent qui sourd de l' intérieur, de la rage et la paix guerrière s' efface ainsi qu' aux premières rafales de l'hiver-dieu que (' hiver est dur mais précède mai)

6. (les appels s'enracinent tiennent a la fécondité des cuivres et du blé nous les enlaçons dans la profondeur des côtes dans la ruche de l'effroi)

7. (mais la foi n' est-elle qu' apprivoisée famille main-d auvre?)

Les accents les plus plaintifs et les plus intimistes du poème se lisent dans les parenthèses. La présence accrue d'un pronom à la première personne ne justifie pas à elle seule la remotivation d'une instance subjective. Le ton élégiaque de certains de ces vers est frappant: mes villes aux pauvres pauvres dans l'aisselle des coffres, musaraignes de la mort, nos os reposent dans leurs chairs... À mi-chemin entre la douleur et la joie, ces plaintes lyriques donnent au poème une tonalité mélancolique, une certaine discrétion dans l'émotion. À bien des égards, l'emploi de la parenthèse accentue la caractère psalmodique du poème. Comme le refrain incantatoire, il crée un espace musical où le ton l'emporte sur le sens (sans l'abolir). La voix dans la parenthèse n'est pas tout à fait celle qu'on entend dans les autres vers; elle provient du même locuteur, mais elle est suffisamment modulée pour être distinctement perceptible. Elle est à la fois la même et l'autre, elle reprend certains motifs du poème à voix basse, en les murmurant. Double voix dont l'effet est encore une fois d'orienter la lecture du poème en invitant le lecteur à s'arrêter sur les signifiants plutôt qu'aux références signifiées.

L'altérité est constitutive au poème et toute sa structure repose sur un jeu de voix qui combine l'homogène et l'hétérogène. En somme, trois voix sont distinctement perceptibles dans le poème: la voix première, la plus marquée dans 
le développement des strophes, la voix du refrain, où l'homophonie est la plus sensible, et la voix mise entre parenthèses. Le texte glisse d'une voix à l'autre, brisant de la sorte le ton univoque du locuteur. Le poème échappe ainsi à l'hégémonie d'une voix totale. Si l'on ne doit pas confondre cette trivocalité avec le polyphonisme bakhtinien (puisque les trois voix relèvent d'un seul sujet énonciateur), elle n'en est pas aussi éloignée que Bakhtine le laisse entendre. Ces trois voix constituent un dialogue intérieur où se manifeste la fragmentation de la conscience du poète: le corps se divise pour le plaisir / et la satisfaction (p. 214), écrit Lapointe au début du poème «Gravitations». Il n'y a pas totalisation des voix multiples, la fragmentation n'est jamais sur le point d'être récupérée dans un tout homogène. Á la base de l'écriture de Lapointe se trouve le désir de parer au danger que veut justement éviter Dostö̈evski par le dialogisme, c'est-à-dire la menace d'un ton unique:

Pour lui (Dostoïevski), les liens stylistiques essentiels ne sont pas ceux qui existent entre les mots dans le cadre d' un seul énoncé monologique, mais les liens dynamiques intenses entre des énoncés, entre des centres discursifs autonomes et indépendants, libérés de la dictature verbale et interprétative d' un style monologique, $d^{\prime}$ un ton unique. ${ }^{45}$

L'expérience de la révolte de terre est en ce sens l'expérience d'un sujet divisé, jamais pareil au même. L'homme, le travailleur manuel, la main, se met à désirer l'autre: prendre possession de son corps, c'est chercher à se multiplier.

C'est ici que l'on arrive au point de carrefour des deux axes intertextuels du langage poétique: le poème de Lapointe ouvre la révolte exprimée par Parti pris à une révolte plus globale, non réductible au programme idéologique de la revue et comparable à la transformation de l'homme proposée par le surréalisme. Cet évasement de la matrice idéologique partipriste se manifeste dans plusieurs lieux du discours poétique. L'homogénéité d'un sujet collectif est excédée par un sujet multiple, morcelé, qui ne se sent jamais tenu de se justifier en tant que sujet ni de se recentrer. La révolte socialiste revendiquée par Parti pris, timide à bien des égards, faite par des intellectuels et en grande partie pour des intellectuels, est lisible dans le poème mais tellement dilatée, tellement ambitieuse qu'elle n'est plus la même - bien qu'elle ne soit définissable qu'en relation avec cette dernière. Il s'agit d'une révolte autre, mais cet autre, si l'on peut dire, n'offre pas la possibilité d'annuler le même. Le surréalisme du poème de Lapointe crée dans ce discours partipriste de l'ouverture, du possible, mais celui-ci impose en revanche à la polysémie du poème certains repères qui en limitent l'extension. Le poème ne déborde pas la revue sur tous les fronts: il y a évasement mais non évasion.

La poésie est une éloquence, selon la formule de Lacoue-Labarthe. Elle se perd dans les mots, dans les beaux mots, elle est l'excès de parole, le débord idéologique, le désir non-fini (infini?):

45 Mikhain Bakhtine, la Poétique de Dostoïevski, Paris, Seuil, 1970, p. 265-266. 
Ce dans quoi sombre la poésie, ce qui la noie, c'est une éloquence. Mais il ne faut pas se méprendre sur l'éloquence : il s'agit bien entendu d' un «trop-dire» mais le «trop» ne signifie pas seulement l'abondance ou la surabondance (le «trop-plein»), il signifie aussi, ou d'abord, l' excès (le «trop-en-dire»). ${ }^{46}$

Traduite dans le poème, la révolte socialiste est là, mais trop, accrue. Lacoue-Labarthe déduirait probablement de ceci qu'elle est prolongée démesurément de telle sorte qu'elle devient impraticable, irréductible à toute finalité extérieure à elle-même. Mais ce «trop» poétique ne se pose pas en tant qu'absolue différence, en tant que pur autre. Aussi excessif soit-il, le langage du poème ne renvoie pas qu'à lui-même. Il se définit précisément dans son point de contact avec les deux textes (Parti pris et le surréalisme), entre la réduction de l'un et l'éclatement de l'autre, entre la révolte directement reproduite et la révolte absolue. Cette trouée du poème dans le dispositif idéologique de Parti pris s'effectue dans des lieux spécifiques de son discours qu'il s'agit en dernière analyse de déterminer.

\section{Une dérive idéologique: le psaume}

S'il est un mot qui ne fait pas partie du vocabulaire courant de Parti pris, c'est bien le mot «psaume». Publier un psaume en 1963 au Québec, en pleine période anticléricale, et dans une revue anticléricale par surcroît, est troublant: pourquoi? Écartons dès maintenant l'explication selon laquelle Lapointe aurait ainsi voulu emprunter ce titre à la littérature sainte pour parodier le discours religieux. Il est sans doute paradoxal que l'auteur du Vierge incendié écrive une prière, mais ce n'est pas en vain qu'il situe son poème dans le champ de l'écriture biblique: celle-ci participe de l'intérieur au poème, fait partie de l'architectonique du texte, dirait Bakhtine. La rhétorique, le ton et même la musique du psaume sont perceptibles. À lui seul, le refrain onze fois proféré rythme le poème à la manière d'une psalmodie, crée une zone de texte en dehors du tumulte des mots, une zone qui permet au lecteur de retirer brièvement son attention aux mots. Le leitmotiv ô psalmodie ô psaumes opère à deux niveaux: d'une part, il désigne la forme biblique du psaume et d'autre part, il fait passer les mots à un niveau qui n'est plus celui de la signification: le niveau de l'incantation magique. Le procédé, fréquent dans toute la poésie, permet au lecteur de répéter les mots pour le seul plaisir de la répétition. L'accumulation d'assonances le distrait peu à peu et l'initie à une sorte d'état de grâce poétique qui rapproche le texte d'une musique. La révolte de terre s'inscrit au cœur de cet envoûtement sonore pendant lequel se transforme l'être humain, comme si le poème parvenait, à force de faire répéter les mêmes mots, à révéler l'homme en tant qu'autre. La poésie incantatoire fait passer l'ordre sonore devant l'ordre de la signification et parvient à faire ce que Parti pris rêve d'accomplir: la libération magique de l'homme, la révolte sur le mode incantatoire. Maheu, parlant de poésie incantatoire, écrit ceci: Plutôt que de se porter témoin de l'événement catharsique

46 Philippe Lacoue-Labarthe, op. cit., p. 40. 
comme en rend compte le récit, elle s'adressera au lecteur comme paroles mêmes de sa propre libération [...]. C'est accepter une rédemption qui est immersion dans la négation de soi pour se dépasser, se retrouver autre. ${ }^{47}$

Le refrain annonce l'événement transformateur mais sans y prendre part directement, inchangé du début à la fin du poème. Le renversement que j'ai analysé plus haut n'atteint pas le refrain parce que c'est le refrain qui semble opérer le renversement. Le refrain instaure le temps du prévisible, de l'intact, de l'inaltérable, mais il introduit concurremment la possibilité d'une expérience différente opposée au quotidien. Non seulement se répète-t-il d'une fois à l'autre, mais il contient, en outre, lui-même la figure de la redondance puisqu'il est constitué de deux mots de la même famille grammaticale et sémantique. Ce procédé est familier à Lapointe qui l'utilise par exemple dans le poème «le temps tombe» ${ }^{48}$. Cet énoncé, repris sept fois dans le poème, est composé comme $\hat{o}$ psalmodie $\hat{o}$ psaumes de deux lexèmes redoublés phoniquement. Dans son analyse de ce dernier poème, Pierre Nepveu décrit le refrain comme suit: La répétition du même est [...] le chant du même, rythme et psaume qui font surgir la différence, l'espace éclaté de l'imaginaire qui se déploie dans les strophes de développement. ${ }^{49}$ Sans reprendre tout le raisonnement de Nepveu, retenons qu'il interprète ce jeu du même et de l'autre comme l'investissement du temps archaïque dans et par le présent socio-culturel de la modernisation. C'est le refrain le temps tombe qui pose le temps de l'actuel, du quotidien, alors que les strophes de développement font intervenir l'imaginaire du passé, l'inconscient archaïque. C'est exactement l'inverse qui se produit dans ô psalmodie ô psaumes: le temps quotidien, celui du travailleur, apparaît dans les strophes, ponctué par l'incantation atemporelle du leitmotiv. Dans les deux poèmes, même osmose des deux niveaux temporels, comme s'ils étaient essentiels l'un à l'autre.

Les interrogations solennelles du poème, le ton de l'invocation et l'adresse finale au «seigneur» appartiennent à la rhétorique du psaume. Mais plus encore que la rhétorique, le vocabulaire du poème dénote constamment le religieux: diluvienne métamorphose, pères crucifiés, anges, dimanche, croix, dieu, terre propice... Par-dessus tout, ce sont les titres Pour les âmes et «Psaume pour une révolte de terre» qui désignent avec le plus d'ostentation une dimension religieuse. Cette dimension est à tel point «inadmissible» pour l'époque (et surtout pour le milieu dans lequel le poème circulait) que certains critiques, dont Suzanne Paradis, se sont sentis embarrassés par elle: Ce titre (Pour les ames) qui m' avait à sa parution curieusement indisposée en me rappelant les troncs des églises ainsi pompeusement justifiés, je ne le lis pas encore sans malaise. ${ }^{50}$ Un malaise

47 Pierre Maheu, cité par André-J. Bélanger, op. cit., p. 159-160.

48 Ce poème de Pour les ámes est construit comme aPsaume pour une révolte de terrex: recours au leitmotiv, propositions indépendantes, parataxe, prépondérance du substantif, emploi important des parenthèses...

49 Pierre Nepveu, «La tombée du temps», Etudes françalses, vol. XVI, $n^{\circ} 2$, avril 1980, p. 54.

50 Suzanne Paradis, «Le Réel absolu de Paul-Marie Lapointex, Livres et auteurs québécots, 1971, p. 124. 
injustifié, rétorquera Nepveu, puisque l'imagerie religieuse de Pour les âmes ne concerne rien d'autre que le rél, bien qu'elle s'efforce de dépasser toute conception platement positive du réel. ${ }^{51}$ Il associe l'inscription du sacré chez Lapointe à une sorte d'anamnèse, une remontée d'un passé que le contexte fébrile de l'époque tend à refouler. La fonction mnémonique du poème revêt ainsi une fonction rituelle.

Écrire un psaume en 1963, c'est appeler un type de discours étrange, difficile à interpréter pour un lecteur de Parti pris. Le poème de Lapointe est chant, appel, invocation, adresse, prière. Il est tout entier tourné vers un destinataire, comme si l'être ne pouvait se concevoir sans ses rapports avec l'autre. C'est en ce sens que se comprend l'inscription de la prière dans le poème. Dans son étude récente de la poésie de Paul Celan, Lacoue-Labarthe analyse un poème intitulé «Psaume» (dans la Rose de personne) et en arrive à une équation fort générale entre prière et poésie:

Qu' un poème maintienne ainsi, à l'extrême limite il est vrai, la possibilité de la prière, ne serait-ce pas le signe qu'il existe un lien, et peutêtre nécessaire, entre prière et poésie? Que la poésie en son essence serait prière et toute prière, à l' inverse, poème ?52

Ce rapprochement entre la prière et le poème repose en grande partie sur la forme «advocative» du poème, sur le fait que le psaume suppose, en ne s'adressant à personne en particulier, en adressant une parole libre, la présence d'un autre, d'un innommé (désigné une fois par le terme «seigneur», ce qui n'autorise certes pas à conclure grand chose, surtout pas qu'il s'agit de Dieu). Mais la prière chez Lapointe n'est pas qu'une métaphore de la poésie: elle inscrit à l'intérieur du projet de révolte partipriste une dérive imprévue.

Dans ses «Notes pour une poétique contemporaine», Lapointe s'est avisé du rapport entre la forme poétique et le jazz et plusieurs critiques en ont fait la clef de l'interprétation de ses textes. Il écrit ceci: La forme d'improvisation particulière au jazz - ad libitum sur une structure donnée, linéaire et verticale - me paraît devoir exprimer de la façon la plus concrète la forme de la nouvelle poésie. ${ }^{53}$ Le plus long poème de Pour les âmes s'appelle d'ailleurs «Blues» et il y cite le nom de Miles Davis: car Miles Davis est un pont d' acier et de chair (p. 254). Présente dans l'explicite du texte, la référence au jazz se lit comme une image poétique. Il n'est pas question de chercher à définir formellement l'inscription du jazz dans le poème, pas plus qu'il n'a été question de définir la notion d'improvisation en poésie. Le jazz est avant tout une référence du poème de Lapointe à un univers musical particulier et il serait téméraire, voire futile. de lui accorder une valeur théorique.

Le mot «jazz» est bien sûr lié à la culture américaine noire dont il évoque la plainte chantée. Il désigne la possible liberté collective et correspond tout à fait

51 Pierre Nepveu, les Mots a l'écoute, op. cit., p. 207.

52 Philippe Lacoue-Labarthe, op. cit., p. 114-115.

53 Paul-Marie Lapointe, «Notes pour une poétique contemporainex, op. cit., p. 88. 
en cela au projet de révolte accompli par le poème. Mais il désigne aussi une pratique artistique contemporaine qui place «Psaume pour une révolte de terre» dans un courant de la modernité. L'univers musical du poème se déploie donc autour de deux pôles qui paraissent au premier abord extrêmement éloignés l'un de l'autre: le jazz et la psalmodie. Considéré de cette façon, le poème se présente sous le signe d'une séduction musicale d'autant plus efficace que les deux formes de musique sont loin d'être incompatibles.

Si le psaume et le jazz semblent se rapporter à des époques fort différentes, l'une ancienne, l'autre moderne, il faut ajouter que le jazz était associé à ses débuts aux manifestations religieuses des Noirs américains. Par ses rythmes élémentaires, son rapport au corps, à la voix, il connote l'irrationnel et le primitif, mais il contient aussi un sème religieux qui le rapproche de la psalmodie. Il est donc à la fois l'expression musicale de la tradition moderne et d'une tradition identifiée au départ à la ferveur religieuse. Il ne fait pas de doute cependant que c'est au nom de sa valeur moderne, au nom de la nouveauté qu'il représente, que le jazz circule dans le discours québécois de l'époque de Parti pris. Patrick Straram va jusqu'à déclarer, dans sa chronique «Jazz dans la vie quotidienne», qu'il manque à tout Canadien français une pratique quotidienne du jazz et il fait du jazz le lieu de toutes les vertus insurrectionnelles. Il cite plusieurs écrivains québécois influencés par cette musique: Michel Garneau... Et Paul-Marie Lapointe. Et Yves Préfontaine. Et Michel Van Schendel. Et quelques autres... Est-ce un hasard si pour tant de poètes d'ici, et les plus révolutionnaires, le jazz est un facteur déterminant dans la vie quotidienne ?54 Sur le plan symbolique, le jazz est équivoque en ce sens qu'il participe de deux traditions (la religion et la modernité) extrêmement méfiantes l'une par rapport à l'autre. Mais le témoignage de Straram, tout naïf qu'il soit, indique bien le privilège qui lui est accordé chez les écrivains québécois modernes. En ce cas, si l'équivoque du jazz fait le jeu de la modernité, comment expliquer la référence affichée au psaume dans le poème de Lapointe?

En 1948, Lapointe publiait, on le sait, un recueil explosif où l'une des cibles de choix était sans conteste l'Église. Quinze ans plus tard, sans volteface ${ }^{55}$, il publie un poème qui se dit un psaume, l'une des formes les plus anciennes de la prière judéo-chrétienne. L'effet est double: d'une part, le psaume évoque un passé extrêmement lointain, délesté du poids d'une histoire apparemment inacceptable; d'autre part, ce passé archaïque est revalorisé par le recours à une autre forme ancienne qui jouit à l'époque où le poème est écrit d'une plusvalue symbolique de la modernité: le jazz. Se télescopent ainsi par-dessus l'histoire des civilisations occidentales l'aujourd'hui et le jadis, c'est-à-dire l'actuel et l'intemporel. Le psaume échappe à la critique anti-religieuse de l'époque partipriste parce qu'il connote un religieux qui n'est pas contaminé par l'histoire.

54 Patrick Straram, «Jazz dans la vie quotidiennew, Parti pris, vol. I, $n^{\circ} 3$, décembre 1963 , p. 57.

55 Paul-Marie Lapointe, «Poésie sociale et morale», dans Guy Robert, op. cit., p. 86. 
Le caractère paradoxal de la juxtaposition du psaume et de la révolte dans le titre du poème ne disparaît pas pour autant: le psaume s'oppose clairement à la révolte telle qu'elle est proposée par Parti pris. Dans la mesure où la révolte de terre se trouve dans la position d'un complément du substantif «psaume», elle n'a pas d'existence autonome, elle n'a pas la possibilité de faire abstraction de la tradition à laquelle renvoie le psaume. Tandis que l'identité québécoise passe pour Parti pris par une authenticité d'abord linguistique (dont le joual est le meilleur exemple), elle se fonde dans le poème de Lapointe sur une tradition nationale qui réclame son héritage religieux. Il ne s'agit pas de vestiges catholiques qui collent à la mémoire du poète malgré son anticléricalisme. Tel est le cas plutôt d'un Maheu qui se plaint encore en 1965 qu'd strictement parler, il n'y a pas un seul athée au Québec. Nous sommes bien quelques-uns à faire de notre mieux pour le devenir, mais si fort que nous essayons, le compte n'y est pas et Dieu ne nous lâche pas. ${ }^{56}$ L'auteur du Vierge incendié écrit «âme» et «psaume» comme s'il cherchait à préserver le souvenir des expériences les plus humiliantes et les plus réprouvées du passé.

Le psaume, comme le jazz, est équivoque puisqu'il est présenté en rapport avec la révolte moderne. Dans un sens, le psaume donne à la révolte des proportions accrues, celle-ci ne pouvant faire l'économie du passé rejeté par Parti pris. Dans un autre sens, la révolte donne à la prière, c'est-à-dire à une constante de la tradition nationale, des enjeux spécifiques qui semblent contredire la continuité requise par la tradition. L'aller-retour entre le psaume et la révolte définit un rapport qui n'est plus strictement d'opposition puisque les deux termes se fécondent mutuellement. Dérive idéologique du discours partipriste, le psaume constitue peut-être ce «trop-en-dire», cet excès de la parole poétique dont parlait plus haut Lacoue-Labarthe. Cette dérive fonctionne donc comme l'interaction de plusieurs discours, comme une réponse aux propositions idéologiques de Parti pris.

\section{Du poétique à l'idéologique}

Tous ces mots (aller-retour, rapport, dérive, interaction, réponse...) suggèrent une certaine structure spécifique du rapport du poétique à l'idéologique. La charge référentielle du poème de Lapointe se modalise de telle sorte que les mots qui désigneraient un univers social québécois ne sont jamais posés en relation directe avec cet univers. On vient de voir que le terme «psaume» ne se réfere pas immédiatement au religieux de 1963, mais à un religieux épuré dont les valeurs dépassent les questions de pratique. Le «réel absolu» comprend la révolte partipriste et le psaume. La référence la plus marquée à un type d'organisation sociale est celle du travailleur, de l'ouvrier dans l'usine. Ce sont eux les protagonistes de la révolte et c'est lors d'une grève que celle-ci se déclenche. Mais la description très populiste de cette grève, véritable mythification de l'ouvrier révolté pancartes en mains avec un cri et des armes (p. 210), désigne

56 Pierre Maheu, «Les fidèles, les mécréants et les autres», Partl pris, vol. II, $n^{\circ} 8$, avril 1965 , p. 20. 
moins les luttes syndicales des années 60 qu'une certaine figure légendaire de l'ouvrier ${ }^{57}$. L'ordre symbolique dans lequel le poème situe ses références à la société québécoise refuse d'être soumis à l'historicité contraignante des dates. Le poème pousse le circonstanciel, le contingent à un niveau symbolique beaucoup plus général, ce qui ne veut pas dire qu'il annule toute forme de représentation commune. En poésie, il n'y a pas d'antinomie véritable entre figuration et défiguration.

Le psaume et l'ouvrier auraient donc ceci en commun qu'ils accèdent par une sorte de lob au-dessus du filet des événements historiques au statut d'archétypes. Du désuet à l'archaïque, du démodé au primitif ou, pour reprendre le titre d'un livre de Marshall McLuhan, Du cliché à l'archétype. En privilégiant la répétition de l'ancien, Lapointe fait advenir le plus qu'ancien et reproduit de la sorte une des contradictions les plus profondes de la conscience moderne: la tradition de la rupture. La modernité choisit de rompre avec la tradition immédiate pour rejoindre un passé immémorial qui est une tradition magnifiée livrée à l'excès de l'absolu.

McLuhan décrit le cliché de cette façon: L'ordinaire de l'existence est fait d' une infinité de gestes et d'environnements que la conscience ne remarque pas parce que leur omniprésence les indifférencie. ${ }^{58}$ Le cliché remotivé par le poème devient archétype au moment où il parvient à la conscience, où il devient utilisé plus qu'usé. Ces termes, poursuit-il, seraient tout à fait interchangeables si à "cliché» ne correspondait pas un temps d' insensibilisation, et d «archétype», un temps de resensibilisation. ${ }^{59}$ En tant que manifestation incantatoire, le poème de Lapointe cherche justement à échapper à sa récupération sous forme de cliché. Pour cette raison, il endort les mots et les écrit en faisant appel à un niveau de perception primaire qui médiatise leur fonction référentielle. Voici comment McLuhan parle du rapport entre le poème et l'archétype:

Lorsque Baudelaire dit de la poésie qu'elle est «une sorcellerie évocatoire», il signale tout simplement qu'il a retrouvé la perception archaïque, «auditive» du langage. Pour l' homme des temps anciens, le langage suscite la réalité d' une façon directe, immanente; c'est une formule magique. Il n'y a pas d' intervalle entre le cliché et l'archétype; l'homme primitif vit dans une conscience archétypale, inclusive. ${ }^{60}$

La révolte chez Lapointe n'est jamais quitte envers le révolu. Plus encore, le poème se donne pour tâche de confronter le révolu et le routinier et espère

57 La semaine est encore de six jours dans le poème (vont aux bois le dimanche, p. 207); la limitation du titre de travailleur aux seuls ouvriers ne correspond pas non plus à la réalité de 1963; enfin, le ton presque ouvriériste du poème paran̂t quelque peu anachronique.

58 Marshall McLuhan, Du cliché à l'archétype. La foire du sens, Montréal, Hurtubise HMH, Paris, Ed. Mame, 1973, p. 130.

59 Ibid.

60 Ibid., p. 131. 
produire, du choc répété des deux moments, un mouvement perpétuel. Par la remotivation constante des clichés sous forme archétypale, le poète récupère un passé en ruines pour se préserver contre sa propre décomposition. Il accomplit très exactement ce qu'Octavio Paz décrit comme la fusion de tous les temps et de tous les espaces en un hic et nunc: l'archaïque s'actualise et le passé immédiat est déjà lointain 61 .

L'histoire littéraire québécoise a toujours éprouvé un malaise devant l'œuvre de Lapointe. Associée d'abord au courant surréaliste, elle semble s'en dégager par la suite. Durant les années de la Révolution tranquille, on ne sait trop de qui rapprocher cette poésie et l'absence de références explicites au pays ou à la nation y est pour quelque chose. Nepveu écrit: $A$ cet égard, il est essentiel de constater que le temps mythique du commencement et de l' identité fondatrice, pas plus que la terre n'est une image de l' enracinement ou de l'élémentaire. Dès lors ce n'est pas par hasard que l'on a tant de mal a situer Pour les ames dans le courant poétique des années 60.62 Pourtant les mots «âmes» et «psaume» situent d'emblée le recueil dans le droit fil de la tradition québécoise puisqu'ils font référence à un axe majeur de la tradition nationale.

Pendant que Parti pris attend la définition de l'identité québécoise, le poème affirme que cette identité est de l'ordre de l'évidence: Du fait même qu'elle existe, ma poésie est québécoise, mais ça va de soi, quoi. ${ }^{63} \mathrm{La}$ «Revendication fondamentale» à laquelle fait référence Lapointe dans ses textes de prose ne récupère pas le passé national mais l'éprouve simultanément avec la modernité contemporaine. La modernité de Lapointe s'autorise d'une tradition que la modernité de la revue abjure. Est-ce à dire que le poème situe la révolte moderne dans une continuité linéaire et aplatie, dans une tradition de la répétition? Non, parce que la tradition ici, comme archétype, est fluide, mobile et se transforme à mesure qu'elle est confrontée aux clichés quotidiens. Celui de la révolte, certes un des lieux communs les plus avérés de la Révolution tranquille, accède dans le poème, en passant par la révolte surréaliste et moderne en général, à ce statut archétypal. Ainsi tradition et révolte s'échangent les signes, chacune devenant tour à tour cliché et archétype. Déployé par delà le contexte idéologique de Parti pris, l'archétype de la révolte de terre se formulerait de la manière suivante: l'accompli n'existe pas, la vraie vie est la révolte.

61 L'époque moderne est celle de l'accélération du temps historique. Je ne veux pas dire, il va de soi, qu'aujourd'hui les années et les jours passent plus rapidement, mais qu'il s'y passe plus de choses. Il s'y passe plus de choses et toutes presque en même temps, non l'une après l'autre, mais simultanément. L'accélération est fusion: tous les temps et tous les espaces convergent en un ici et un maintenant. (Octavio Paz, Point de convergence, Paris, Gallimard, coll. «les Essais», 1976, p. 19).

62 Pierre Nepveu, \&La tombée du temps», loc. cit., p. 61.

63 Paul-Marie Lapointe et Robert Melançon, «L'injustifiable poésie», Etudes françaises, vol. XVI, n 2 , avril 1980 , p. 95. 